\title{
PROMOVER LA HABILIDAD CIENTÍFICA DE OBSERVACIÓN EN EDUCACIÓN PRIMARIA:UNA PERSPECTIVA DESDE LA REFLEXIÓN PEDAGÓGICA
}

\section{Promoting the Scientific Skill of Observation in Elementary Education: A Perspective from the Pedagogic Reflection}

\section{Promover a habilidade de observação científica no ensino fundamental: uma perspectiva da reflexão pedagógica}

\author{
Paola Andrea Pérez Narváez* \\ Yulieth Nayive Romero Rincón ${ }^{* *}$ \\ Francisco Javier Bernal Sarmiento ${ }^{\star \star}$
}

\begin{abstract}
Resumen
El presente escrito surge de una reflexión pedagógica propia que emerge de la problematización de la práctica en relación con el fortalecimiento de la habilidad científica de observación en los estudiantes de educación primaria del Colegio San Cayetano en la ciudad de Bogotá. De esta manera, se expone cómo la práctica pedagógica promueve la observación, señalándose las fortalezas y dificultades identificadas. De igual forma, se especifican los cambios que se han implementado luego de realizar el proceso de reflexión, tales como la utilización del contexto situacional, lingüístico y mental para el abordaje de la observación, y la visibilización de las observaciones a través de rutinas de pensamiento para la evaluación formativa de esta habilidad. Se concluye indicando que la reflexión pedagógica se convierte en el eje motor de los cambios en la práctica y por tanto puede favorecer una transformación pedagógica para fortalecer las habilidades científicas en los estudiantes de primaria.
\end{abstract}

Palabras clave: reflexión de la práctica pedagógica; observación; educación primaria; habilidades científicas

* Estudiante de Maestría en Pedagogía de la Universidad de la Sabana. Licenciada en Biología por la Universidad Distrital Francisco José de Caldas. Docente adscrita a la Secretaría de Educación Distrital. Correo electrónico: paolapn2015@gmail.com;

** Estudiante del Doctorado Interinstitucional en Educación, Universidad Pedagógica Nacional. Magíster en Pedagogía de la Universidad de la Sabana. Docente adscrita a la Secretaría de Educación Distrital. Correo electrónico: yuliethrori@unisabana.edu.co;

*** Magíster en Educación de la Universidad de la Sabana. Docente del Colegio Marymount en Bogotá. Correo electrónico: francisco.bernal@unisabana.edu.co 


\section{Abstract}

This paper comes from an own pedagogical reflection, which emerged of making problematic the teaching practice regarding the strengthening of the scientific skill of observation in basic primary students of San Cayetano School located in Bogota City. Thus, there are strengths and difficulties exposed that are presented in the pedagogical practice in the promotions of this skill, as well as, the changes that have been implemented after the identification of the mentioned strengths and weaknesses. To conclude, it is indicated that the pedagogical reflection turns into the main axis of the changes in the practice, that here are framed in the categories of contextualization and make visible students' observations.

Keywords: pedagogical practice reflection; observation; primary education; scientific abilities

\section{Resumo}

Este artigo surge de uma reflexão pedagógica própria, que emerge da problematização da prática em relação ao fortalecimento da capacidade científica de observação em estudantes do ensino fundamental do Colégio San Cayetano, na cidade de Bogotá. Dessa forma, expõe-se como a prática pedagógica promove a observação, indicando os pontos fortes e as dificuldades identificadas. Da mesma forma, são especificadas as mudanças implementadas após a realização do processo de reflexão, como o uso do contexto situacional, linguístico e mental para a abordagem da observação e a visibilidade das observações através de rotinas de pensamento para a avaliação formativa dessa habilidade. Conclui-se indicando que a reflexão pedagógica se torna a força motriz das mudanças na prática e, portanto, pode favorecer uma transformação pedagógica para fortalecer as habilidades científicas em alunos do ensino fundamental.

Palavras chave: reflexão da prática pedagógica; observação; ensino fundamental; habilidades científicas 


\section{Introducción}

El reto actual de la educación en ciencias es aportar a la formación de ciudadanos que sean capaces de "comprender y actuar sobre un mundo rápidamente cambiante y profundamente impregnado por la ciencia y la tecnología" (Declaración de Budapest, citada por Furman, 2016, p. 23). En este marco, la educación en ciencias le ha apostado al desarrollo del pensamiento científico como eje articulador de conocimientos, habilidades y actitudes que les permitan a los ciudadanos tomar decisiones justificadas y responsables en y para su entorno.

De este modo, se le ha atribuido a la escuela, y en especial al docente de ciencias, la responsabilidad de formar en conceptos, habilidades y actitudes científicas. Dentro de estas, las habilidades científicas han recobrado importancia en los primeros años de escolaridad. Al respecto, Di Mauro, Furman y Bravo (2015) mencionan que en la educación primaria se sientan las bases del pensamiento científico de las personas, de manera que "mucho del éxito o fracaso de su aprendizaje futuro en el área de ciencias naturales dependerá de estos primeros años de formación" (Di Mauro et ál., 2015, p. 3).

En este contexto se ha determinado que, durante las primeras edades, la educación en ciencias ofrezca espacios donde los niños exploren y potencien sus habilidades de pensamiento científico (Daza y Quintanilla, 2011). De este modo, al terminar el proceso escolar, los estudiantes tendrían unas habilidades científicas básicas para desenvolverse en cualquier ámbito de su vida.

Sin embargo, se ha venido señalando que "el ciudadano promedio no tiene, en general, una suficiente formación científica de base" (Secretaría de Educación Distrital [SED], 2007, p. 56), de modo que carece de conocimientos y habilidades científicas (Claxton, 1994, citado por Ortiz y Cervantes, 2015), lo cual puede evidenciarse en los bajos desempeños de los estudiantes en las pruebas PISA, Terce y Saber.

Al respecto, Ortiz y Cervantes (2015) mencionan que "ha faltado compromiso y rigor en las instituciones educativas para desarrollar proyectos investigativos que promuevan procesos y habilidades científicas en los niños" (p. 16). Por tanto, pensar en el fortalecimiento de las habilidades científicas implica empezar a reflexionar sobre las prácticas pedagógicas que se llevan a cabo. De acuerdo con Calvo (2002), el docente "no se puede quedar en el nivel esquemático y descriptivo de lo que pasa en el aula. Es necesario interpretar esa realidad para superar lo puramente fenoménico, aquello que en la realidad aparece disperso" (p. 74). Desde este punto de vista, la reflexión pedagógica se convierte en una necesidad y oportunidad para interpretar lo que sucede en el aula.

Dentro de este marco, reflexionar pedagógicamente implica comprender los fundamentos teóricos de la práctica y promover la coherencia entre la teoría expuesta (lo que los docentes dicen que hacen y creen) y la teoría en uso (lo que realmente hacen) (Larrive, 2008). De esta manera, la reflexión pedagógica permite la identificación de aciertos y limitaciones en la práctica para posibilitar cambios en ella.

Teniendo en cuenta estas consideraciones, con este artículo se busca mostrar cómo la reflexión pedagógica llevada a cabo por la docente de Ciencias Naturales de primaria (grados cuarto y quinto) del Colegio San Cayetano de Bogotá ha sido promotora de cambios orientados al fortalecimiento de la observación, una habilidad básica en el inicio de procesos científicos e investigativos (Romero y Pulido, 2015) y fundamental para el desarrollo de otras habilidades, como la comparación y la clasificación (De Sánchez, 1998).

\section{Desarrollo}

A partir de la perspectiva de reflexión pedagógica señalada por Larrive (2008), a continuación se presenta una reflexión sobre cómo la práctica pedagógica promovía la habilidad de observación. Enseguida se exponen los cambios que se han venido implementando a partir de las fortalezas y dificultades identificadas.

Para comenzar, es importante indicar cómo se entiende la observación. Siguiendo lo establecido por la Organización de las Naciones Unidas para la Educación, la Ciencia y la Cultura (Unesco), se trata de una habilidad de pensamiento científico en la que se examina "un objeto o fenómeno directamente con los sentidos o a través de instrumentos apropiados, para conocer su estado en un momento, comportamiento o cambios en el tiempo" (2016, p. 18).

Desde esta perspectiva, se evidencia que la práctica pedagógica promovía procesos de observación en los cuales los estudiantes detallaban plantas del entorno e imágenes sobre procesos de reproducción. Se describían los colores de las plantas y los cambios en estas después de someterlas a experimentos. Por otro lado, a partir de las secuencias de imágenes, los estudiantes debían responder una serie de preguntas orientadas a lo que estaba sucediendo en estas y realizaban conteos de algunos aspectos.

De este modo, la práctica promovía procesos de observación que se realizaban desde el sentido de la visión, considerando algunas veces aspectos cuantitativos. Sin 
embargo, dejaba de lado la generación de espacios que promovieran la observación desde la utilización de otros sentidos para identificar aspectos como sonidos, olores, sabores, consistencias, durezas, componentes, etc., lo cual, de acuerdo a Santelices (1989, citado en Romero y Pulido, 2015), es característico de una buena observación.

Ahora bien, los procesos de observación que proponía la práctica pedagógica eran generalmente contextualizados. Así, los estudiantes observaban plantas de su entorno y secuencias de imágenes que evidenciaban procesos reproductivos de animales que conocían. Al respecto, Furman (2016) indica que esta contextualización de los aprendizajes despierta en el estudiante su interés por aprender y ayuda a que comprenda el sentido que tienen los aprendizajes en su vida real.

No obstante, aunque las actividades orientadas por la docente invitaban a la observación contextualizada, las planeaciones y evaluaciones no reflejaban esta habilidad. Por el contrario, en ellas se manifestaban únicamente los conceptos biológicos y procesos del entorno vivo y físico que se estipulan en los Estándares básicos de competencias en Ciencias Naturales (Ministerio de Educación Nacional [MEN], 2006).

Esta situación indica que la práctica pedagógica estaba promoviendo una enseñanza de carácter empirico-positivista, en concordancia con Gallego y Pérez (1999), porque focalizaba la enseñanza en la presentación de conceptos, que se enseñaban como verdades establecidas que los estudiantes debían memorizar.

Esta concepción de la enseñanza de las ciencias también demostraba unos fines propedéuticos por parte de la docente. Al priorizar los contenidos conceptuales en lugar de los procedimentales (habilidades de pensamiento científico, como la observación), se reflejaba una preferencia por los primeros, quizá por la visión aún arraigada de que en la escuela "debe orientarse a la selección de conceptos que servirán de base para los estudios superiores" (Meinardi, 2010, p. 16).

En conclusión, la práctica pedagógica generaba espacios para que los estudiantes realizaran observaciones contextualizadas, principalmente desde el uso del sentido de la vista. Sin embargo, desde lo propuesto por los autores, es necesario que en el fortalecimiento de esta habilidad se abarque el uso de todos los sentidos. De este modo, se hizo patente la necesidad de abrir espacios donde se promovieran observaciones profundas y visibles, para que los estudiantes lograran dialogar sobre estas y la docente pudiera hacer un seguimiento de los avances en los procesos de observación.

\section{Cambios implementados}

A partir de la anterior reflexión y teniendo en cuenta que la habilidad de observación se puede mejorar (Santelices, 1989, citado en Romero y Pulido, 2015), se han empezado a proponer una serie de cambios fundamentados en la teoría y la práctica, a fin de fortalecer esta habilidad en los estudiantes. A continuación se describen dichos cambios, desde las categorías de la contextualización y la visibilización de las observaciones.

\section{El contexto en los procesos de observación}

La práctica pedagógica sigue promoviendo la observación del entorno y de imágenes en los que se muestran fenómenos conocidos por los estudiantes. Sin embargo, ahora lo hace explícito desde la planeación. Así, el conocimiento contextual por parte del profesor, un componente de su conocimiento profesional (Valbuena, 2007), se convierte en la base orientadora que le permite diseñar, implementar y evaluar los procesos de observación.

Por consiguiente, a la hora de planear las observaciones que los estudiantes van a realizar, la docente tiene en cuenta las características socioculturales, ambientales, institucionales e históricas que conciernen al contexto situacional (Bermúdez y De Longhi, 2012). Esto para que los procesos de observación sean interesantes y llamativos, los aprendizajes significativos $y$, consecuentemente, se generen preguntas que den paso a la construcción de nuevos conocimientos.

Sin embargo, además del contexto situacional, durante la planeación también se tiene presente el contexto lingüístico y mental. El primero se relaciona con el habla de los profesores y alumnos, y con la terminología propia del contenido y su lógica (Bermúdez y De Longhi, 2012). En este sentido, el contexto lingüístico puede ser el propio de la ciencia y la tecnología, que se refiere a las habilidades de pensamiento científico, en este caso la observación, así como el lenguaje del contenido que concierne a los conceptos involucrados en las clases. De este modo, se busca que el docente use con frecuencia el lenguaje científico, dentro del cual se encuentra la palabra observación.

Por su parte, el contexto mental está conformado por todo lo "no observable", como las representaciones y referentes sobre el tema (Bermúdez y De Longhi, 2012). Para el caso de la observación, la tendencia es hacia el nivel "Sin categorizar" (sc, de acuerdo a la adaptación hecha por Romero y Pulido [2015] de lo propuesto por Santelices [1989]), con un porcentaje del $73 \%$. Teniendo en cuenta esta tendencia, se proponen procesos de observación que lleven a los estudiantes a desarrollar niveles más altos. 
En resumen, la práctica pedagógica sigue promoviendo procesos de observación desde el contexto situacional, pero ahora también lo hace desde el contexto lingüístico y mental de los estudiantes. Por tanto, la práctica pedagógica no solo debe conocer el entorno sociocultural, ambiental, institucional e histórico de los estudiantes, sino también el lenguaje científico pertinente para la clase y los procesos mentales que desarrollan los estudiantes, con el fin de proponer prácticas que les permitan desarrollar mejores procesos de observación.

\section{Visibilizar las observaciones de los estudiantes}

En atención a que las observaciones no eran socializadas y se restringían únicamente a los estudiantes que las realizaban, se ha buscado que estas ahora se hagan visibles para darlas a conocer. En este sentido, se han utilizado rutinas de pensamiento, entendidas como "procedimientos, procesos o patrones de acción que se utilizan de manera repetitiva parar manejar y facilitar el logro de las metas o tareas específicas" (Ritchhart, Church y Morrison, 2014, p. 85), que permitan a los estudiantes hacer procesos de observación, organizar la información y facilitar su comunicación.

Esta visibilización de las observaciones, a través de rutinas de pensamiento, ha permitido a la docente realizar diagnósticos sobre sus estudiantes, tal como lo indican Tishman y Palmer (2005). Estos diagnósticos los ha usado para diferentes fines, por ejemplo, como un punto de partida para abordar una nueva observación y promover una evaluación formativa sobre estas (Furman, 2016).

Este último aspecto ha sido determinante para favorecer que los procesos de observación sean cada vez más profundos. Es así que la práctica pedagógica reconoce asertivamente las observaciones realizadas por los estudiantes y hace recomendaciones para que estas se vuelvan más profundas. De este modo, la visibilización de las observaciones permite al docente aportar de manera formativa al proceso de observación.

Por otro lado, la visibilización de las observaciones ha contribuido a que los estudiantes comprendan que el conocimiento no es un cúmulo de significados hechos por otros que deben memorizarse, sino que, por el contrario, es un producto que deriva de múltiples observaciones que son constantemente debatidas con otros. De esta manera, la visibilización de las observaciones ha favorecido una cultura en la que el estudiante se convierte en el protagonista de la construcción de su conocimiento.
En resumen, la visibilización de las observaciones ofrece a los estudiantes oportunidades para involucrarse en procesos de observación profundos. Sin embargo, para ello se hace necesario que en las prácticas pedagógicas se diseñen e implementen de manera continua actividades diferentes. De aquí la importancia de las rutinas de pensamiento como una oportunidad que además de dejar a la vista lo observado ofrece estructuras a los estudiantes para organizar y comunicar sus observaciones.

\section{Conclusiones}

La reflexión pedagógica permitió comprender que la observación era una habilidad que estaba siendo abordada durante las clases de ciencias naturales de primaria (grados cuarto y quinto). Sin embargo, también se identificó que los procesos de observación promovidos no se estaban realizando de una manera constante ni profunda. En consecuencia, la reflexión pedagógica derivó en la proposición de cambios paulatinos, a partir de la contextualización del aprendizaje y la visibilización de las observaciones.

A partir de la categoría del contexto, la práctica pedagógica empezó a utilizarlo como base orientadora para planear, implementar y evaluar los procesos de observación. Así, se retomó el contexto situacional para proponer procesos que fueran interesantes para los estudiantes de primaria; el contexto lingüístico para generar oportunidades de habla desde las ciencias; y el contexto mental para promover observaciones más profundas.

Finalmente, con relación a la visibilización de las observaciones, la práctica pedagógica implementó rutinas de pensamiento que las pusieran de manifiesto. En consecuencia, se propusieron estructuras que buscaban facilitar su organización para favorecer su comunicación y permitir al docente realizar una evaluación formativa de los procesos de enseñanza.

Por todo lo anterior, se puede concluir que la reflexión pedagógica permitió la identificación de fortalezas y debilidades de la práctica de promoción de la habilidad científica de observación en la básica primaria, lo que dio lugar a la proposición de cambios específicos orientados a fortalecerla. Por lo tanto, se puede afirmar que los procesos de reflexión se convierten en el eje de la práctica pedagógica, porque facilitan su transformación para fortalecer las habilidades científicas en los estudiantes de primaria. 


\section{Referencias}

Bermúdez G. y De Longhi, A. L. (2012). El conocimiento didáctico de contenidos biológicos de Ecología. En R. C. Flores (coord.), Experiencias Latinoamericanas en educación ambiental (pp. 17-31). Monterrey: CEC Y TE NL-CAEIP.

Calvo, G. (2002). El docente: responsable de la investigación pedagógica. En M. Osorno (ed.), Experiencias docentes, calidad y cambio escolar: investigación e innovación en el aula (pp. 72-83). Memorias del v Congreso Distrital de Investigación Educativa e Innovación Pedagógica IDEP, Bogotá, Colombia.

Daza, S. y Quintanilla, M. (2011). La enseñanza de las ciencias naturales en las primeras edades. Barrancabermeja, Colombia: Litodigital.

De Sánchez, M. (1998). Desarrollo de habilidades de pensamiento. México: Trillas.

Furman, M. (2016). Educar mentes curiosas: la formación del pensamiento científico y tecnológico en la infancia. Buenos Aires: Fundación Santillana.

Gallego, R. y Pérez, R. (1999). Aprendibilidad, enseñabilidad y educabilidad en las ciencias experimentales. Educación y Pedagogía, 25, 87-117.

Larrive, B. (2008). Development of a Tool to Assess Teachers' Level of Reflective Practice. Reflective Practice, 3, 341-360.

Di Mauro, M., Furman, M. y Bravo, B. (2015). Las habilidades científicas en la escuela primaria: un estudio del nivel de desempeño en niños de $4 .^{\circ}$ año. Revista Electrónica de Investigación en Educación en Ciencias, 2, 1-10.

Meinardi, E. (2010). El sentido de educar en ciencias. En E. Meinardi, L. González, A. Revel y M. Plaza, Educar en ciencias (pp. 11-39). Argentina: Paidós.
Ministerio de Educación Nacional (MEN). (2006). Estándares básicos de competencias en Lenguaje, Matemáticas, Ciencias y Ciudadanas. Bogotá: autor.

Organizacion de las Naciones Unidas para la Educación, la Ciencia y la Cultura (Unesco) (2016). Aportes para la enseñanza de las ciencias naturales. Chile: autor.

Ortiz, G. y Cervantes, M. (2015). La formación científica en los primeros años de escolaridad. Panorama, 17, 10-23.

Ritchhart, R., Church, M. y Morrison, K. (2014). Hacer visible el pensamiento (trad. M. Barrera y P. León). Argentina: Paidós.

Romero, Y. y Pulido, G. (2015). Incidencia de las rutinas de pensamiento: observar y preguntar en los estudiantes de grado cuarto, ciclo II del Colegio Rural José celestino Mutis. Memoria para otorgar el título de Magíster en Pedagogía, Universidad de la Sabana, Chía, Colombia.

Secretaría de Educación Distrital. (2007). Orientaciones curriculares para el campo de Ciencia y Tecnología. Bogotá: autor.

Tishman, S. y Palmer, P. (2005). Pensamiento visible [versión electrónica]. Leadership Compass. http:// vidarte.weebly.com/uploads/5/1/5/4/5154246/pensamiento_visible.pdf.

Valbuena, E. (2007). El conocimiento didáctico del contenido biológico: estudio de las concepciones disciplinares y didácticas de futuros docentes (tesis doctoral inédita). Universidad Complutense de Madrid, España. 